\title{
Coordination in Price Setting and the Zero Lower Bound: A Global Games Approach
}

\author{
Mitsuru Katagiri* \\ mkatagiri@imf.org
}

* Research and Statistics Department (currently International Monetary Fund)

Papers in the Bank of Japan Working Paper Series are circulated in order to stimulate discussion and comments. Views expressed are those of authors and do not necessarily reflect those of the Bank.

If you have any comment or question on the working paper series, please contact each author.

When making a copy or reproduction of the content for commercial purposes, please contact the Public Relations Department (post.prd8@boj.or.jp) at the Bank in advance to request permission. When making a copy or reproduction, the source, Bank of Japan Working Paper Series, should explicitly be credited. 


\title{
Coordination in Price Setting and the Zero Lower Bound: A Global Games Approach*
}

\author{
Mitsuru Katagiri ${ }^{\dagger}$
}

August 2016

\begin{abstract}
In this paper, I construct a two-period general equilibrium model and describe price competition among monopolistically competitive firms as a coordination game. While the model has multiple equilibria with different levels of inflation (positive or zero), the equilibrium selection in line with global games implies that the economy with a high natural interest rate, i.e., high expected productivity growth, tends to move into the equilibrium with positive inflation. The policy analyses indicate that monetary policy measures such as an increase in the target inflation rate and a decrease in the lower bound of nominal interest rates can prevent the economy from moving into the zero inflation equilibrium even in the face of low expected productivity growth.
\end{abstract}

Keywords: Inflation Indeterminacy; Effective Lower Bound; Global Games JEL Classification: D82; E31; E52

\footnotetext{
*The author is grateful for comments from Kosuke Aoki, Tomohiro Hirano, Michihiro Kandori, Yuichi Yamamoto, and colleagues at the Bank of Japan, as well as participants of seminars at the University of Tokyo. Any remaining errors are the sole responsibility of the author. The views expressed in this paper are those of the author and do not necessarily reflect the official views of the International Monetary Fund and the Bank of Japan.

${ }^{\dagger}$ Research and Statistics Department, Bank of Japan (currently International Monetary Fund, Email: mkatagiri@imf.org)
} 


\section{Introduction}

Most central banks in advanced economies set a positive level for target inflation and conduct monetary policy to keep the inflation rate around the target. It is assumed that, in the process of realizing positive inflation, firms raise prices together through implicit coordination via relative price adjustments. While such implicit price coordination resulting in positive inflation represents one possible equilibrium in terms of price competition among firms, another possible equilibrium is one in which firms do not dare to raise prices because other firms also do not raise prices, and as a result zero inflation is realized. This implies that strategic complementarity in firms' price setting behavior may potentially lead to multiple equilibria with different levels of inflation. Therefore, an important question for policy makers, particularly in advanced economies that have faced prolonged low inflation, is what conditions or policies are necessary to achieve coordination among firms together with a positive level of inflation.

In this paper, I describe price competition among firms as a coordination game in a general equilibrium model and discuss what conditions or policies are necessary for realizing a positive level of inflation. I first discuss the possibility that the effective lower bound on nominal interest rates (ELB) causes multiple equilibria with different levels of inflation (an inflation equilibrium and a zero-inflation equilibrium) under strategic complementarity in price competition among monopolistically competitive firms. I then apply the equilibrium selection principle in line with global games to the model with multiple equilibria by adding a small amount of noise to private signals about productivity growth. The model with uncertainty (i.e., the model in which private signals about productivity growth contain a small amount of noise) indicates that, as a result of strategic behavior of agents, the equilibrium selection takes place based on the expected productivity growth rate, which corresponds to the natural interest rate in the model. This result implies that the economy with low economic growth tends to reach the ELB and consequently experience low inflation in the long run.

The result that low economic growth leads to low inflation in the long run is, however, not widely shared in the recent literature on monetary economics. For example, in 
standard New Keynesian models, long-run inflation has essentially nothing to do with economic growth and is determined only by the target inflation rate. Monetary policy makers, on the other hand, often express the view - particularly in the context of the limited effectiveness of conventional monetary policy at the ELB - that low inflation is related to a low natural interest rate. ${ }^{1}$ Since a low natural interest rate in the long run corresponds to low expected growth, this view essentially implies that monetary policy makers regard inflation to be closely linked to expected economic growth. This intuitive view among central bankers finds some support in empirical observation. Figure 1 plots the average GDP growth rate and the average inflation rate over the last two decades (1996-2015) for OECD countries. The figure indicates that there possibly is a positive correlation between economic growth and inflation in the long run, which I try to explain in this paper from a theoretical point of view. In that sense, the result in this paper can be interpreted as providing one potential mechanism underlying policymakers' intuition as well as empirical evidence on the long-run relationship between economic growth and inflation.

While this study takes the expected growth rate as an exogenous variable and argues that equilibrium selection between the inflation equilibrium and the zero-inflation equilibrium is based on the expected growth rate, the policy analysis in this paper implies that the central bank can prevent the economy from moving into the zero-inflation equilibrium through appropriate policy actions even in the face of low economic growth. For example, the policy analysis indicates that the central bank can decrease the threshold of the growth rate for equilibrium selection by raising the target inflation rate and/or lowering the ELB by introducing a negative interest rate policy. This result of the policy analysis implies that if the prolonged low inflation recently observed in developed countries corresponds to the zero-inflation equilibrium in the model, the central bank can escape from such an equilibrium by adopting appropriate policy measures.

\footnotetext{
${ }^{1}$ For instance, Kuroda (2016) argues that "[t]he [second] major challenge that made it more difficult to overcome deflation is the decline in the natural rate of interest reflecting a deceleration in Japan's growth potential. [....] Given these challenges, Japan was unable to find an appropriate cure for the chronic disease of prolonged deflation. This is how a deflationary equilibrium took hold."
} 
Figure 1: Inflation and output growth for OECD countries

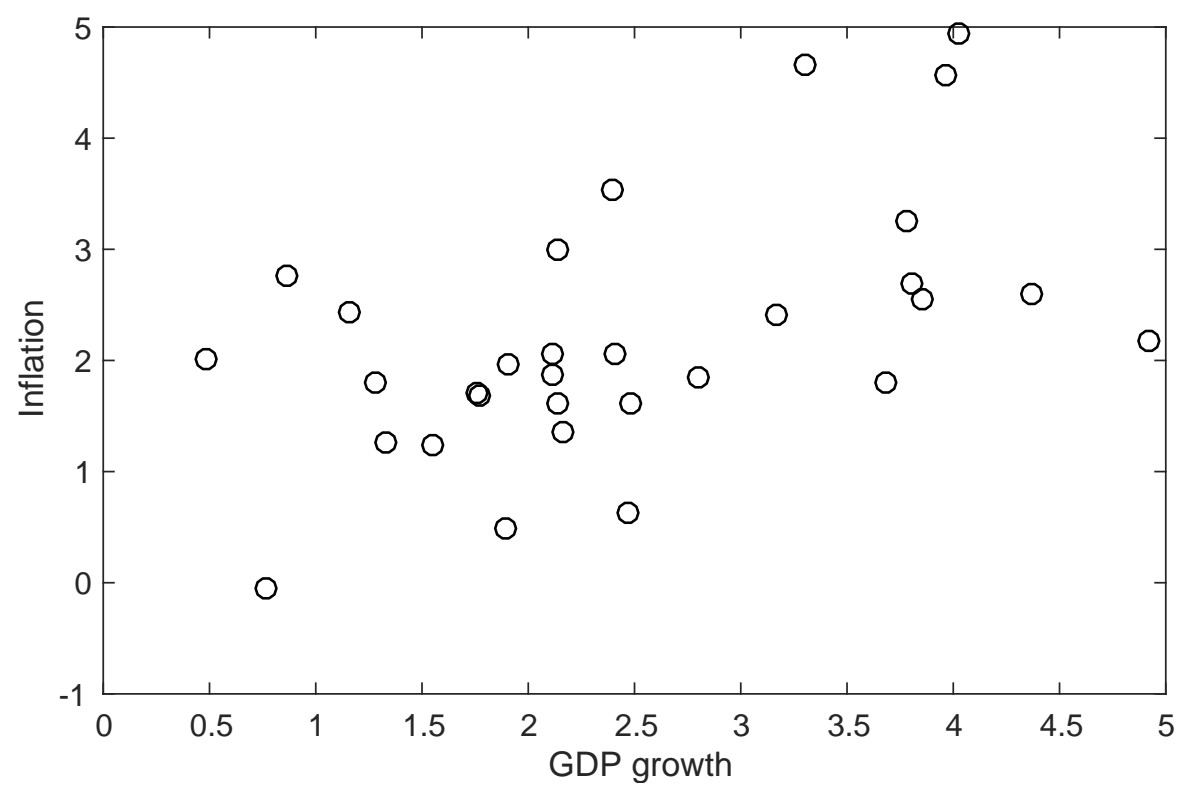

Note: The figure plots the average GDP growth rate and the average inflation rate over the last two decades (1996-2015) for OECD countries. Inflation is calculated in terms of the year-on-year change in the consumer price index for all items excluding food and energy. For some countries (Chile, Estonia, and Slovenia), the observation period is less than 20 years due to data limitations. In addition, Mexico, Hungary, and Turkey are excluded here as outliers, since these three countries experienced hyperinflation in the late 1990s. 
This paper contributes to the literature on the application of global games to macroeconomic issues. Morris and Shin (1998) discuss equilibrium selection in a currency crisis in relation to the market value of a currency, while Goldstein and Pauzner (2005) discuss equilibrium selection in a bank run in relation to the return on assets. In a similar vein, this paper discusses equilibrium selection between multiple equilibria with different levels of inflation in relation to the expected productivity growth rate. Moreover, this paper is also related to the literature on inflation indeterminacy. In the model with the ELB, inflation is undetermined and has multiple equilibrium values, as discussed by Benhabib et al. (2002). While this paper adopts a backward looking model like Taylor (1999) rather than a forward looking model as Benhabib et al. (2002) do, it can be shown that the logic behind the multiplicity of equilibria is very similar in the two models. The analysis in this study therefore provides a useful intuition to understand equilibrium selection between multiple equilibria with different levels of inflation. Finally, this study is related to the literature providing policy analyses at the ELB (e.g., Christiano et al. (2011), Fernandez-Villaverde et al. (2014) and Iacoviello and Michelis (2016)). A distinctive

difference from previous studies is that while they examine policy effects by assuming that inflation eventually returns to the target level, this study discusses policy effects on the equilibrium selection between multiple equilibria.

The paper proceeds as follows. In Section 2, I construct a simple two-period general equilibrium model and describe price competition among firms as a coordination game. Section 3 characterizes the equilibrium for the model without uncertainty and indicates the possibility of multiple equilibria. Section 4 discusses equilibrium selection in the model with uncertainty, and conducts some policy analyses regarding the relationship between inflation and monetary policy. Finally, Section 5 provides concluding remarks.

\section{Model}

The model is a simple two-period general equilibrium model. The private sector of the economy consists of a representative household, consumption-good firms, and a continuum of intermediate-good firms. The central bank conducts monetary policy using 
nominal interest rates as the policy tool and follows the Taylor rule with an effective lower bound on nominal interest rates (ELB). Each agent's behavior is described in turn.

\section{$2.1 \quad$ Household}

The representative household supplies labor to obtain wage income $W_{t} L_{t}$, where $W_{t}$ denotes the nominal wage and $L_{t}$ denotes hours worked. In addition, because the household owns all firms in the economy as a stockholder, it also receives dividends $D_{t}$ as another source of income. The household allocates its income to consumption, $c_{t}$, and savings, the latter of which take the form of a nominal one-period bond, $B_{t}$. The household's budget constraint for $t=1$ and 2 is,

$$
P_{t} c_{t}+B_{t}=R_{t-1} B_{t-1}+W_{t} L_{t}+D_{t}
$$

where $P_{t}$ is the price level and $R_{t}$ is the nominal interest rate. Here it is assumed that $B_{0}=0$. The household's budget constraint is rewritten in real terms by dividing it by $P_{t}$ :

$$
c_{t}+b_{t}=\frac{R_{t-1}}{\pi_{t}} b_{t-1}+w_{t} L_{t}+d_{t}
$$

where $\pi_{t} \equiv P_{t} / P_{t-1}$ is the gross inflation rate and real variables are denoted by lower-case letters. Since the initial price level is normalized to one $\left(P_{0}=1\right)$, the inflation rate in period one is equal to the price level $\left(\pi_{1}=P_{1}\right)$. The household's inflation expectation is formed in a completely adaptive and backward-looking manner:

$$
E_{1}\left[\frac{1}{\pi_{2}}\right]=\frac{1}{\pi_{1}}
$$

The household maximizes the sum of utility for $t=1$ and 2 by choosing its consumption and labor supply,

$$
\max _{c_{t}, L_{t}} E_{1} \sum_{t=1}^{2}\left[\log c_{t}-\psi L_{t}\right]
$$

subject to the budget constraint (2) and the inflation expectation (3). The first order conditions for $c_{t}, B_{t}$ and $L_{t}$ yield the Euler equation,

$$
\frac{1}{c_{1}}=E_{1}\left[\frac{R_{1}}{\pi_{2}} \frac{1}{c_{2}}\right]
$$


as well as the labor supply function,

$$
\frac{w_{t}}{c_{t}}=\psi
$$

\section{$2.2 \quad$ Firm}

The representative consumption-good firm produces the final good, $y_{t}$, by aggregating intermediate goods $y_{i, t}$, using the constant elasticity of substitution (CES) aggregator $y_{t}=\left(\int_{0}^{1} y_{i, t} \frac{\theta-1}{\theta} d i\right)^{\frac{\theta}{\theta-1}}$, where $\theta>1$ is the elasticity of substitution. Let $p_{i, t}$ be the price of each intermediate good. The price index, $P_{t}$, is then defined as

$$
P_{t}=\left(\int_{0}^{1} p_{i, t}^{1-\theta} d i\right)^{\frac{1}{1-\theta}}
$$

and the demand for each intermediate good is derived as a result of profit maximization of the representative consumption-good firm,

$$
y_{i, t}=\left(\frac{p_{i, t}}{P_{t}}\right)^{-\theta} y_{t}
$$

A continuum of intermediate-good firms produces differentiated intermediate goods using labor $l_{i, t}$, subject to the following linear technology:

$$
y_{i, t}=A_{t} l_{i, t}
$$

where $A_{t}$ is aggregate productivity in period $t$. Here, $A_{1}$ is normalized to one (i.e., $A_{1}=1$ ) and $A$ is then defined as $A_{2}=A_{2} / A_{1} \equiv A$. That is, $A$ is the expected productivity growth from period 1 to 2 . For the time being, it is assumed that $A$ is perfectly forecastable and common knowledge among firms. Later, a small amount of noise will be added to $A$, which plays a crucial role in the equilibrium selection.

Under monopolistic competition, intermediate-good firm $i$ maximizes its profits by setting the price of its differentiated products subject to the menu cost for price changes, $\xi$. Hence, intermediate-good firm $i$ chooses its prices, $p_{i, t}$, so as to maximize the profit function $\Pi\left(p_{i, t} ; c_{t}, w_{t}, P_{t}, A\right)$, which is defined as

$$
\Pi\left(p_{i, t} ; c_{t}, w_{t}, P_{t}, A\right) \equiv\left[\frac{p_{i, t}}{P_{t}} y_{i, t}-w_{t} l_{i, t}-\mathbb{I}_{\left\{t=1 \& \frac{p_{i, t}}{p_{i, t-1}} \neq 1\right\}} \xi\right]
$$


subject to (7), (8), and the market clearing condition, $y_{t}=c_{t}$. Here, the initial price level for each firm $i$ is assumed to be one $\left(p_{i, 0}=1\right)$ for all firms. ${ }^{2}$ Note that menu costs $\xi$ apply only when the firm changes the price in period 1. In other words, all prices are completely flexible in period 2. Also note that expected productivity growth $A$ is the relevant variable for the optimization problem in period 1 as well as that in period 2, because it influences $c_{2}$ and as a result $c_{1}$ via the Euler equation (4). In particular, a high $A$ leads to a high $c_{2}$ and $c_{1}$, thus increasing the incentive for raising prices. ${ }^{3}$ As a result of the intermediate-good firms' optimization, the optimal pricing strategy is defined as

$$
p_{i, t}^{*}\left(c_{t}, w_{t}, P_{t}, A\right)=\arg \max _{p_{i, t}} \Pi\left(p_{i, t} ; c_{t}, w_{t}, P_{t}, A\right)
$$

Since the initial price level for each firm $i$ is equal to one $\left(p_{i, 0}=1\right)$, inflation and the price level in period 1 are equal, $\pi_{i, 1}=p_{i, 1}$, and can be used interchangeably in period 1 . Therefore, when analyzing price competition in period 1 , the optimal strategy for inflation $\pi_{i, 1}^{*}\left(c_{1}, w_{1}, P_{1}, A\right)=\arg \max _{\pi_{i, 1}} \Pi\left(\pi_{i, 1} ; c_{1}, w_{1}, P_{1}, A\right)$ instead of price level $p_{i, 1}^{*}\left(c_{1}, w_{1}, P_{1}, A\right)$ is used for the analysis hereafter.

\subsection{Central Bank}

The central bank sets the nominal interest rate $R_{1}$ according to the following Taylor rule with an $\operatorname{ELB}(\kappa)$,

$$
R_{1}=\max \left[A \bar{\pi}\left(\frac{\pi_{1}}{\bar{\pi}}\right)^{\phi}, \kappa\right],
$$

where $\bar{\pi}$ is the target inflation rate and $\phi$ is the responsiveness to inflation. The Taylor rule basically indicates that the nominal interest rate is equal to the neutral interest rate $A \bar{\pi}$ times the response to the inflation gap.

The max function indicates that the central bank sets the nominal interest rate to $\kappa$ if the nominal interest rate based on the Taylor rule is lower than $\kappa$. In the literature, the

\footnotetext{
${ }^{2}$ This assumption implies that there is no price dispersion in period 0 . This is consistent with the assumption of no heterogeneity across firms in this model.

${ }^{3}$ The mechanism that high expected productivity growth leads to an incentive for raising current prices is similar to the mechanism described in Fernandez-Villaverde et al. (2014).
} 
ELB is usually set to one; however, with some central banks having introduced negative interest rate policies, it may be necessary to set the ELB lower than one. Section 4 considers the ELB as a policy variable and examines the effects of lowering the ELB.

\section{Equilibrium without Uncertainty}

This section characterizes the equilibrium for the model described in the previous section. In particular, I focus on how the inflation rate in period $1, \pi_{1}$, is determined in equilibrium as a result of firms' strategic pricing decisions. Note that since there is no uncertainty regarding expected productivity growth $A$, it is perfectly forecastable and common knowledge across firms. The case without this assumption will be discussed in Section 4 .

\subsection{Best Response Function and Nash Equilibrium}

In order to characterize the equilibrium, the model is solved by backward induction: the model is solved for period 2 first and then for period 1 given the equilibrium values for period 2. Since prices are completely flexible in period 2, firms' optimization yields the real wage in period $2, w_{2}=A(\theta-1) / \theta$, and the labor supply function (5) and the production function (8) then yield consumption, $c_{2}=A(\theta-1) /(\theta \psi)$, and the labor supply, $l_{2}=(\theta-1) /(\theta \psi)$, in equilibrium. Note that since all real variables are determined irrespective of the price level $P_{2}$ in the flexible price model, the price level and inflation in period $2, P_{2}$ and $\pi_{2}$, are undetermined in the model. Consequently, in period 1 , firms essentially face a static optimization problem.

Given the equilibrium values in period 2, it can be shown that all macroeconomic state variables in period 1 can be expressed as a function of inflation, $\pi_{1}$. The macroeconomic state variables relevant for firms' pricing decision in period 1 are $\left(c_{1}, w_{1}, \pi_{1}\right)$ as shown in (9). Since (3) and (11) indicate that both $E_{1}\left[1 / \pi_{2}\right]$ and $R_{1}$ are functions of $\pi_{1}$, the Euler equation (4) implies that $c_{1}$ can be expressed as a function of $\pi_{1}$ given the equilibrium value of $c_{2}$. Finally, with the equilibrium value of $c_{1}$ as a function of $\pi_{1}$, the labor supply 
function (5) gives the real wage $w_{1}$ as a function of $\pi_{1}$.

Once the state variables $\left(c_{1}, w_{1}, \pi_{1}\right)$ are expressed as functions of $\pi_{1}$, the profit function for each firm (9) in period 1 is rewritten as

$$
\tilde{\Pi}\left(\pi_{i, 1} ; \pi_{1}, A\right) \equiv \Pi\left(p_{i, 1} ; c_{1}, w_{1}, P_{1}, A\right) .
$$

Note that $p_{i, 1}$ is replaced by $\pi_{i, 1}$ because of the normalization $p_{i, 0}=1$ for all $i$. Since the inflation rate in period 1 is defined as $\pi_{1}=\left(\int_{i \in[0,1]} \pi_{i, 1}^{1-\theta} d i\right)^{\frac{1}{1-\theta}}$ by $(6)$, and is not influenced by each firm's pricing decision $\pi_{i, 1}$ due to the assumption that each firm has measure zero, the profile of other firms' pricing strategy, i.e., $\left(\pi_{j, 1}\right)_{j \neq i}$, can be replaced by aggregate inflation $\pi_{1}$. Therefore, the best response function for firm $i$ in period 1 is expressed as a function of $\pi_{1}$,

$$
\pi_{i, 1}^{*}\left(\pi_{1} ; A\right)=\arg \max _{\pi_{i, 1}} \tilde{\Pi}\left(\pi_{i, 1} ; \pi_{1}, A\right)
$$

and the symmetric Nash equilibrium for price competition in period 1 is defined as strategy $\pi_{1}^{E}(A)$ satisfying $\pi_{1}^{E}(A)=\pi_{i, 1}^{*}\left(\pi_{1}^{E}(A) ; A\right)$.

\subsection{Multiple Equilibria}

Figure 2 shows the best response functions for firm $i$ as well as the Nash equilibria for price competition in period 1 for the case of $A=1.002$ and $\bar{\pi}=1.02 .{ }^{4}$ In the figure, the horizontal axis represents the inflation rate in period $1, \pi_{1}$, and the vertical axis represents firm $i$ 's optimal pricing strategy $\pi_{i, 1}^{*}\left(\pi_{1} ; A\right)$ in response to inflation $\pi_{1}$. The figure shows the best response functions for the following three cases: (a) an economy without the ELB but with menu $\operatorname{costs}(\kappa=-\infty$ and $\xi>0$, represented by the thin dashed line), (b) an economy with the ELB but without menu costs $(\kappa=1$ and $\xi=0$, thick dashed line), and (c) an economy with the ELB and menu costs $(\kappa=1$ and $\xi>0$, thick bold line). Since the focus here is on symmetric Nash equilibria only, the Nash equilibria for each case are indicated by the intersections of the best response function and the 45 degree line.

\footnotetext{
${ }^{4}$ This example means that the expected productivity growth rate is $0.2 \%$ and the target inflation rate is $2.0 \%$. The other parameters are set to conventional values, which are shown in Table 1.
} 
Figure 2: Best response functions for $\bar{\pi}=1.02$

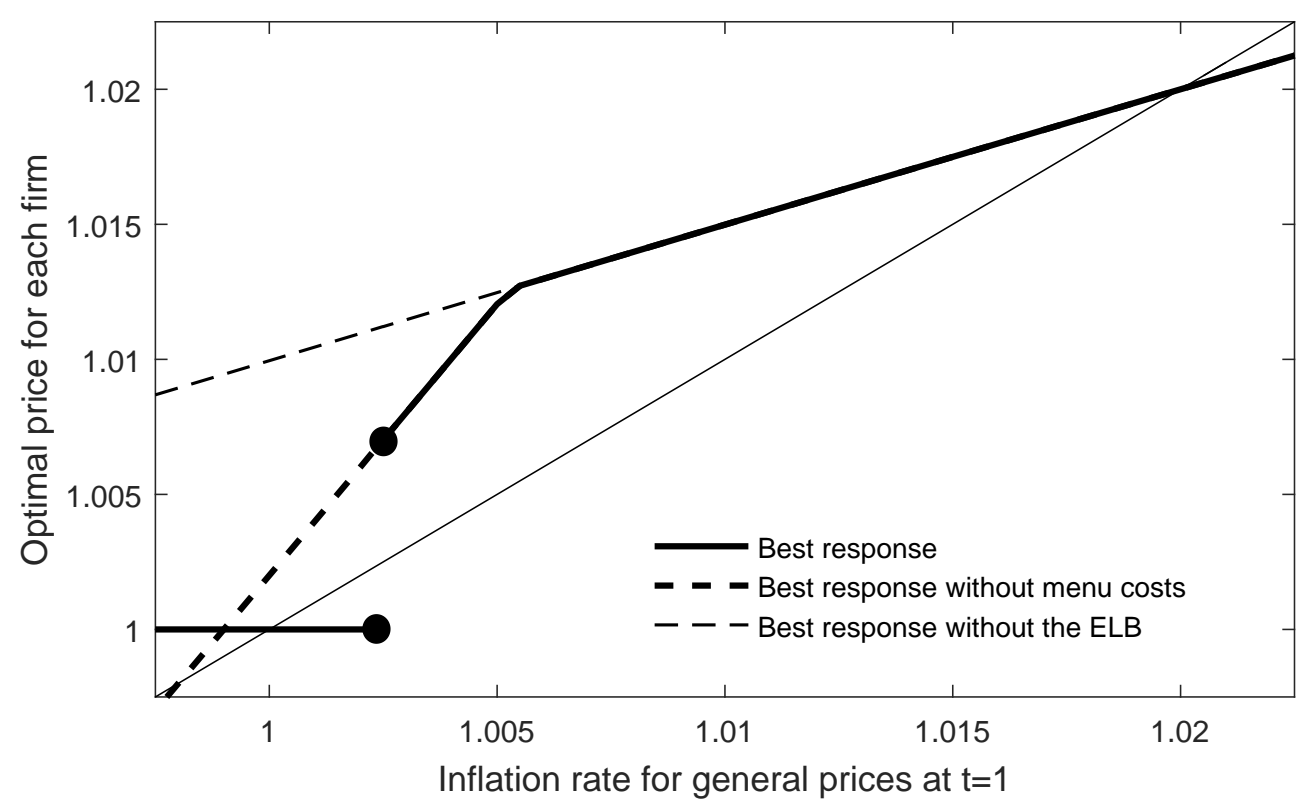

Let us start with the economy without the $\operatorname{ELB}(\kappa=-\infty$ and $\xi>0$, thin dashed line). The figure indicates that the best response function in this economy is always above (below) the 45 degree line for all $\pi_{1}>\bar{\pi}\left(\pi_{1}<\bar{\pi}\right)$, and consequently there is only one Nash equilibrium at $\pi_{1}^{E}=\bar{\pi}$. In order to understand this result intuitively, consider the case of $\pi_{1}<\bar{\pi}$. Since the inflation rate is lower than the target level, the central bank decreases the nominal interest rate below its neutral level $\left(R_{1}<A \bar{\pi}\right)$ according to the Taylor rule in (11). Since this policy reaction of the central bank lowers the real interest rate, $E_{1}\left[R_{1} / \pi_{2}\right]$, and increases aggregate consumption, $c_{1}$, via the Euler equation (4), the incentive for each firm to decrease its price, $\pi_{i, 1}$, is mitigated. As a result, each firm accepts an increase in its relative price, which implies that in this case firms' optimal pricing strategy is to always set their price higher than $\pi_{1}$. That is,

$$
\pi_{i, 1}^{*}\left(\pi_{1} ; A\right)>\pi_{1} \text { for all } \pi_{1}<\bar{\pi}
$$

In the same manner, we have $\pi_{i, 1}^{*}\left(\pi_{1} ; A\right)<\pi_{1}$ for all $\pi_{1}>\bar{\pi}$, thus leading to the only Nash equilibrium at $\pi_{t}=\bar{\pi}$. Menu costs do not affect the best response function unless they are very large, because the optimal price is sufficiently high for the benefits of price 
changes to outweigh menu costs. This result for the economy without the ELB implies that, if the economy is never constrained by the ELB, the central bank can maintain the inflation rate at its target level by appropriately adjusting nominal interest rates and controlling aggregate demand. Therefore, when applying the model to an economy far from the ELB, we do not have to be careful about strategic complementarity in price competition among firms as in a standard New Keynesian model.

Next, let us examine the economy with the ELB (i.e., $\kappa=1$ ). The thick dashed line represents an economy with the ELB but without menu costs $(\kappa=1$ and $\xi=0)$, and the thick bold line represents an economy with both the ELB and menu costs $(\kappa=1$ and $\xi>0$ ). Figure 2 shows that the best response function in these economies has a kink somewhere at $\pi_{1}<\bar{\pi}$ and its slope becomes steeper when $\pi_{1}$ is below the kink. Consequently, it has another intersection with the 45 degree line at $\pi_{1}=1 / A$ for the economy without menu costs (thick dashed line) and at $\pi_{1}=1$ for the economy with menu costs (thick bold line). The best response function in the economy with menu costs (thick bold line) jumps to $\pi_{i, 1}=1$ at some point in a low inflation environment and becomes flat for inflation below this point, because firms lose the incentive to make small price changes given the menu costs. The kink in the best response functions is caused by the ELB, and its location corresponds to the point at which nominal interest rates reach the ELB. The best response function is steeper than the 45 degree line when the economy is constrained by the ELB, because the central bank cannot lower nominal interest rates to deal with a decline in inflation, and consequently cannot increase $c_{1}$ as in the economy without the ELB. That is, when the economy is constrained by the ELB, a decline in $\pi_{1}$ leads to a vicious cycle where

$$
\pi_{1} \downarrow \Rightarrow E_{1} \pi_{2} \downarrow \Rightarrow R_{1} / \pi_{2} \uparrow \Rightarrow c_{1} \downarrow \Rightarrow \pi_{i, 1}^{*} \downarrow
$$

and each firm consequently has an incentive to decrease its price more than the initial decline in $\pi_{1}$, which implies that the best response function is steeper than the 45 degree line when nominal interest rates are constrained by the ELB.

In summary, in the economy with the ELB and menu costs, there are two symmetric Nash equilibria, an inflation equilibrium $\left(\pi_{1}=\bar{\pi}\right)$ and a zero-inflation equilibrium $\left(\pi_{1}=\right.$ 
1). This result is in line with Benhabib, Schmitt-Grohe, and Uribe's (2002) argument that models with the ELB have multiple equilibria with different values of inflation. While the model developed here is backward looking rather than forward looking like their model, the logic underlying the multiplicity of equilibria is very similar: in addition to an inflation equilibrium, the economy has a zero-inflation equilibrium, since the central bank cannot lower nominal interest rates at the ELB to deal with deflationary pressure. Since both equilibria satisfy the equilibrium condition $\pi_{1}^{E}=\pi_{i, 1}^{*}\left(\pi_{1}^{E} ; A\right)$, it is not possible to make a prediction which of the two equilibria will prevail.

\section{Equilibrium Selection and Policy Analysis}

This section discusses the equilibrium selection between the inflation equilibrium $\left(\pi_{1}=\right.$ $\bar{\pi})$ and and the zero-inflation equilibrium $\left(\pi_{1}=1\right)$ characterized in the previous section and then investigates the policy implications. While the previous section showed that there are possibly two symmetric Nash equilibria with different levels of inflation, it is well-known that the multiplicity of equilibria depends on the information structure of the model. In particular, the global game literature suggests that a tiny amount of noise in economic fundamentals leads to equilibrium selection in a model with strategic complementarity. If we can choose one equilibrium out of multiple equilibria, this allows us to use the model for policy analyses by considering the policy effects on the threshold of equilibrium selection. The following considers how a small amount of noise in expected productivity growth $A$ leads to equilibrium selection in the price competition described in the previous section, and conducts some policy analyses regarding the relationship between inflation and monetary policy.

\subsection{Symmetric Bayesian Nash Equilibrium}

In order to discuss equilibrium selection in line with global games, the following modifications to the model are made. First, while in the previous section expected productivity growth $A$ was assumed to be perfectly forecastable and common knowledge among firms, 
this information structure is modified so that (i) expected productivity growth $A$ is drawn from the uniform distribution $U[\underline{B}, \bar{B}]$, and (ii) each firm $i$ receives in period 1 a noisy signal $a_{i}$ following the uniform distribution $U[A-\varepsilon, A+\varepsilon]$. Note that the posterior belief with signal $a_{i}$ is given by

$$
\left\{\begin{array}{ll}
U\left[\underline{B}, a_{i}+\varepsilon\right] & \text { if } a_{i}<\underline{B}+\varepsilon \\
U\left[a_{i}-\varepsilon, a_{i}+\varepsilon\right] & \text { if } \underline{B}+\varepsilon \leq a_{i} \leq \bar{B}-\varepsilon . \\
U\left[a_{i}-\varepsilon, \bar{B}\right] & \text { if } a_{i}>\bar{B}-\varepsilon
\end{array} .\right.
$$

Second, firms' action space in period 1 is not a continuum of prices but limited to a binary set, $\{\bar{\pi}, 1\}$. That is, each firm $i$ in period 1 faces the option of increasing the price at the target inflation rate, $\pi_{1}=\bar{\pi}$, or leaving the price unchanged, $\pi_{1}=1$. The second modification is of course a drastic simplification, but this simplified approach is a reasonable first step to understand the equilibrium selection between multiple equilibria with different levels of inflation. Since the action space is limited to a binary set, menu costs are not needed and thus are set to zero, $\xi=0$. As a result of these two modifications, the strategy for each firm $i$ in period $1, \pi_{1, i}$, should be formulated as a mapping from the signal to the binary action space,

$$
\pi_{1, i}:[A-\varepsilon, A+\varepsilon] \rightarrow\{\bar{\pi}, 1\}
$$

It is easily shown that if the variance of $\varepsilon$ is zero and thus the value of $A$ is common knowledge among firms, the same multiple equilibria are obtained in the modified model with the binary action space. Therefore, the question is which strategy $\pi_{1, i}(\cdot)$ forms a symmetric Bayesian Nash equilibrium in the model with a noisy signal.

Let $0 \leq n \leq 1$ be the fraction of firms which choose $\pi_{i, 1}=\bar{\pi}$. Then, inflation in period $1, \pi_{1}$, can be formulated as a monotonically increasing function of $n$,

$$
\pi_{1}(n) \equiv\left[n \bar{\pi}^{1-\theta}+(1-n)\right]^{\frac{1}{1-\theta}}
$$

for all $\bar{\pi}>1$. Also, the difference between firms' profit when they increase their price, $\pi_{1}=\bar{\pi}$, and when they leave their price unchanged, $\pi_{1}=1$, is defined as a function of $n$ and $A$,

$$
u(A, n) \equiv \tilde{\Pi}\left(\bar{\pi}, \pi_{1}(n) ; A\right)-\tilde{\Pi}\left(1, \pi_{1}(n) ; A\right)
$$


where $\tilde{\Pi}(\cdot)$ is the profit function given by (12). That is, given the values of $n$ and $A$, increasing the price, $\pi_{i, 1}=\bar{\pi}$ (keeping the price unchanged, $\pi_{i, 1}=1$ ), is the optimal choice for a firm if and only if $u(A, n)>0(u(A, n)<0)$.

To ensure the model has reasonable properties, the following assumption is made.

Assumption 1 The parameter values satisfy the following two conditions:

$$
\begin{aligned}
\frac{\bar{\pi}^{\theta-1}-1}{\bar{\pi}^{\theta}-1} \frac{\theta}{\theta-1} & <\bar{\pi}^{\phi-2}<1, \\
A & >\frac{\kappa \bar{\pi}^{\phi-1}}{2} .
\end{aligned}
$$

Condition (13) implies that the reaction of nominal interest rates to inflation, $\phi$, in the Taylor rule (11) should not be too weak or too strong. For example, when $\bar{\pi}=1.02$ and $\theta=6$, the reaction parameter in the Taylor rule should satisfy $\phi \in(1.49,2.0)$, which is consistent with conventional values in the literature. Also condition (14) implies that the expected growth rate should be higher than a certain value, which depends on the target inflation rate. This condition is trivially satisfied in the real economy because when $\bar{\pi}=1.02, \kappa=1.0$, and $\phi=1.5$, this condition requires that $A>0.505$, which means that the expected productivity growth rate should be above $-49.5 \%$. When these conditions are satisfied, the following two lemmas are obtained.

Lemma 1 Assume condition (13) is satisfied. Then, $u(A, n)$ is increasing with respect to $A$ and $n$.

The proof is provided in the Appendix. Intuitively, $u(A, n)$ is increasing with respect to $A$ for the following two reasons. First, a higher $A$ raises the neutral interest rate $A \bar{\pi}$ in (11) and consequently lowers the probability of reaching the ELB. Second, a higher $A$ mitigates the decline in consumption $c_{1}$ at the ELB, because the Euler equation (4) indicates that $c_{1}$ is almost proportional to $c_{2}$ when $R_{1}$ is fixed at $\kappa$. In other words, a higher $A$ mitigates the expansion of the real interest rate gap, $R_{1} / \pi_{2}-A$ at the ELB. On the other hand, $u(A, n)$ is increasing with respect to $n$ because of strategic complementarity among firms in price competition: if more firms choose to raise prices and consequently inflation $\pi_{1}$ increases, firms have a greater incentive to raise their prices in order to avoid a change in relative prices. 
Lemma 2 There exists $\bar{A}$ and $\underline{A}$ satisfying

$$
\begin{aligned}
& u(A, n)>0 \text { for all } n \in[0,1] \text { and } A \geq \bar{A}, \\
& u(A, n)<0 \text { for all } n \in[0,1] \text { and } A \leq \underline{A} .
\end{aligned}
$$

The proof is provided in the Appendix. This lemma implies that if expected productivity growth $A$ is common knowledge and higher (lower) than a certain value, choosing $\pi_{i, 1}=\bar{\pi}\left(\pi_{i, 1}=1\right)$ is the dominant strategy for firm $i$. The economic intuition for this lemma is as follows. For the upper bound $\bar{A}$, it can be shown that the ELB is never binding for all $n \in[0,1]$ when $A$ is higher than a certain value. Therefore, as long as condition (13) ensures that the reaction of nominal interest rates to inflation, $\phi$, is not too small, the central bank can appropriately adjust $R_{1}$, so that firms have an incentive to choose $\pi_{i, 1}=\bar{\pi}$ rather than $\pi_{i, 1}=1$ even when $n$ is small or $\pi_{1}$ is low. For the lower bound $\underline{A}$, on the other hand, it can be shown that the ELB is always binding for $n \in[0,1]$ when $A$ is lower than a certain value. Since the Euler equation (4) implies that consumption $c_{1}$ at the ELB is proportional to $A$, firms eventually lose the incentive to raise prices as $A$ becomes lower and lower.

Given these properties of $u(A, n)$ in Lemma 1 and 2 , the following theorem is obtained in line with the global game literature.

Theorem 1 Let $A^{*}$ be expected growth $A$ solving the equation $\int_{0}^{1} u(A, n) d n=0$. Assume that (i) conditions (13) and (14) are satisfied, and (ii) $\underline{B}<\underline{A}-2 \varepsilon$ and $\bar{B}>\bar{A}+2 \varepsilon$ are satisfied. Then, as $\varepsilon \rightarrow 0$, the threshold strategy, $\pi_{i, 1}\left(a_{i}\right)=\bar{\pi}$ for all $a_{i}>A^{*}$ and $\pi_{i, 1}\left(a_{i}\right)=1$ for all $a_{i}<A^{*}$, forms a unique symmetric Bayesian Nash equilibrium.

The proof is given in the Appendix. Theorem 1 argues that equilibrium selection takes place based on productivity growth $A$ and the threshold is determined as the productivity growth rate at which the expected net profit from raising prices is zero with a uniform prior for $n$. This theorem therefore implies that low economic growth leads to low inflation in the long run. While there is some empirical evidence providing support for this theorem relating long-run inflation to the economic growth rate and monetary policy makers, as mentioned in the introduction, often make this link, it is not 
Table 1: Calibration values

\begin{tabular}{cc}
\hline Parameter & Value or target \\
\hline Monetary policy rule, $\phi$ & 1.5 \\
Elasticity of substitution, $\theta$ & 6.0 \\
Target inflation, $\bar{\pi}$ & 1.02 \\
Effective lower bound, $\kappa$ & 1.0 \\
Labor disutility, $\psi$ & $l_{2}=0.33$ \\
\hline
\end{tabular}

commonly encountered in the monetary economics literature. For instance, in standard New Keynesian economics, long-run inflation has nothing to do with the expected growth rate and is determined only by the target inflation rate set by the central bank. Against this background, the theorem presented here can be interpreted as providing one potential mechanism underlying policymakers' intuition as well as the empirical evidence on the long-run relationship between economic growth and inflation.

\subsection{Quantitative Analysis}

Given the equilibrium selection according to the threshold of expected productivity growth $A^{*}$ established in Theorem 1 , this subsection presents some quantitative analyses and policy experiments regarding the relationship between inflation and monetary policy. In what follows, the model parameters are calibrated and then the threshold $A^{*}$ is computed by solving the model numerically.

The calibration values are shown in Table 1. The responsiveness of nominal interest rates to inflation, $\phi$, and the elasticity of substitution, $\theta$, are set to $\phi=1.5$ and $\theta=6.0$, which satisfy condition (13). The target inflation rate, $\bar{\pi}$, and the value of the ELB, $\kappa$, are set to $\bar{\pi}=1.02$ and $\kappa=1.0$. These policy parameters will be changed later in a policy experiment in order to investigate the effect of policy changes on the threshold $A^{*}$. Finally, the parameter for labor disutility, $\psi$, is chosen so that the labor supply satisfies $l_{2}=0.33$. Under these calibrated parameter values, the threshold value of expected productivity growth $A^{*}$ is computed by numerically solving $\int_{0}^{1} u(A, n) d n=0$ for $A$. 
Figure 3 shows $u(A, n)$ (the difference in firms' profits when they raise their prices and when they leave prices unchanged) with respect to $n$ (the fraction of firms who choose $\left.\pi_{i, 1}=\bar{\pi}\right)$ under three different values of $A(A=1.00,0.993$, and 0.985$)$. There are several notable features in the figure. First, there is a kink in $u(A, n)$ and the slope becomes considerably steeper when $n$ is smaller than the point of the kink. As in the economy without uncertainty shown in Figure 2, the kink is caused by the existence of the ELB: since the central bank cannot address low inflation by lowering nominal interest rates at the ELB, firms lose the incentive to choose $\pi_{i, 1}=\bar{\pi}$ when fewer firms raise their price (i.e., when $n$ decreases). Second, as suggested by Lemma 1, Figure 3 shows that $u(A, n)$ is increasing with respect to both $A$ and $n$. Note, however, that $u(A, n)$ is constant with respect to $A$ if the economy is not constrained by the ELB (i.e., if $n$ is larger than the point of kink). Therefore, the value of $A$ does not influence firms' pricing strategy if the economy is not constrained by the ELB. Given that $u(A, n)>0$ for all $A>\bar{A}$ as suggested by Lemma 3, the figure indicates that the economy will always be in the inflationary equilibrium, $\pi_{1}=\bar{\pi}$, if $A$ is so high that the economy is never constrained by the ELB. In other words, the possibility for reaching the ELB induces the problem of equilibrium selection between the inflation equilibrium and the zero-inflation equilibrium.

Figure 3 shows graphically how $\int_{0}^{1} u(A, n) d n=0$ can be solved for $A$. In the figure, $\int_{0}^{1} u(A, n) d n=0$ means that the size of the area above zero is equal to that below zero. Therefore, when $A=0.993$ (thick bold line), then $u(A, n)$ satisfies $\int_{0}^{1} u(A, n) d n=0$, but when $A>0.993(A<0.993)$, then $\int_{0}^{1} u(A, n) d n>0\left(\int_{0}^{1} u(A, n) d n<0\right)$, meaning that $A=0.993$ is the threshold for the equilibrium selection between the inflation equilibrium and the zero-inflation equilibrium. That is, based on the baseline calibration values, if expected productivity growth is higher (lower) than $-0.7 \%$, the economy is in the inflation equilibrium (zero-inflation equilibrium). 
Figure 3: Net profit when firms raise their prices

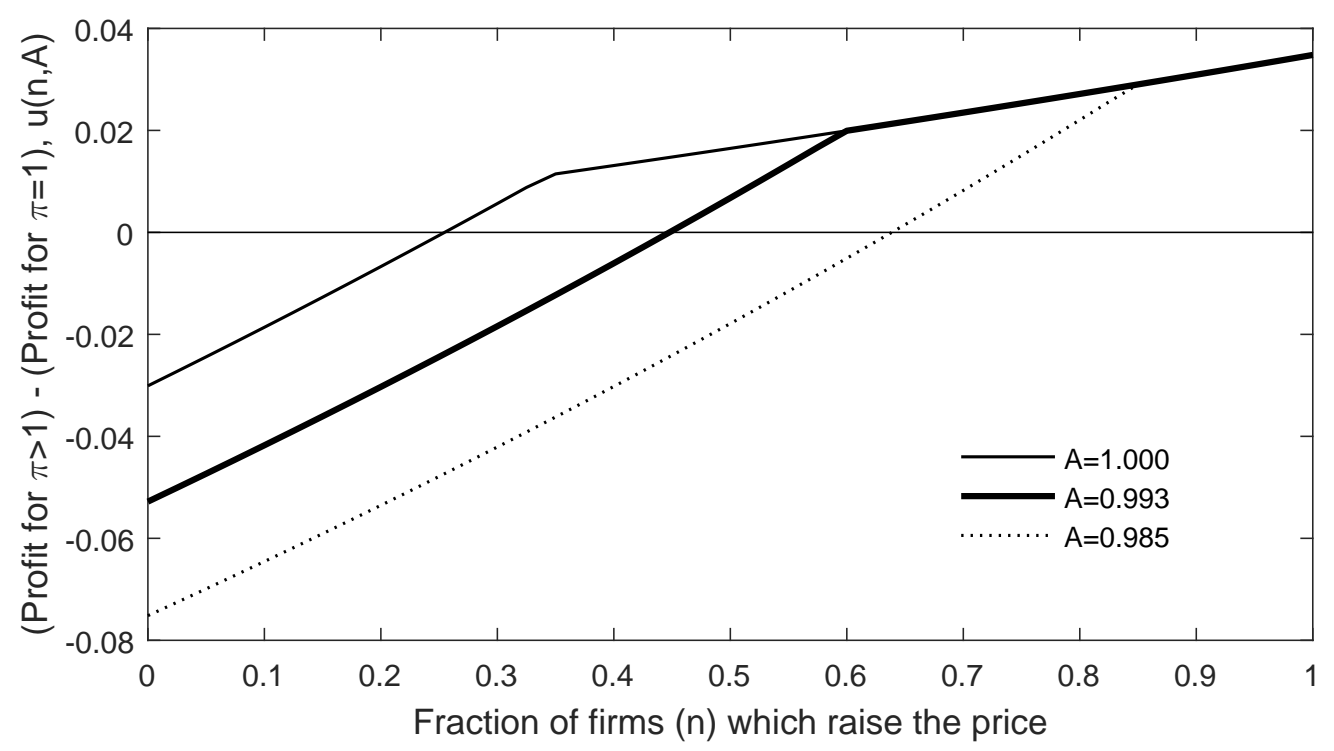

\subsection{Policy Experiment}

This subsection investigates the effects of policy changes on the productivity growth threshold for equilibrium selection between the inflation equilibrium and the zero-inflation equilibrium. For this purpose, several values for (i) the target inflation rate, $\bar{\pi}$, and (ii) the ELB, $\kappa$, are chosen, and the effects of changes in the values on the threshold of equilibrium selection, $A^{*}$, are then examined using comparative statics.

The left panel of Figure 4 shows the result of policy experiments for the target inflation rate, $\bar{\pi}$. In the figure, the horizontal axis represents the value of $\bar{\pi}$, while the vertical axis depicts the productivity growth threshold, $A^{*}$. The figure indicates that the threshold productivity $A^{*}$ is a decreasing function of target inflation $\bar{\pi}$, which implies that the central bank can prevent the economy from moving into the zero-inflation equilibrium even in the face of low expected productivity growth by raising the target inflation rate. For instance, the figure indicates that the central bank can reduce the productivity growth threshold from $-0.7 \%$ to $-1.6 \%$ by raising the target inflation rate from $2 \%$ to 4\%. Since in this model inflation expectations are assumed to be formed in a purely backward looking manner, the rise in target inflation provides stimulus to the economy 
Figure 4: Results of policy experiments
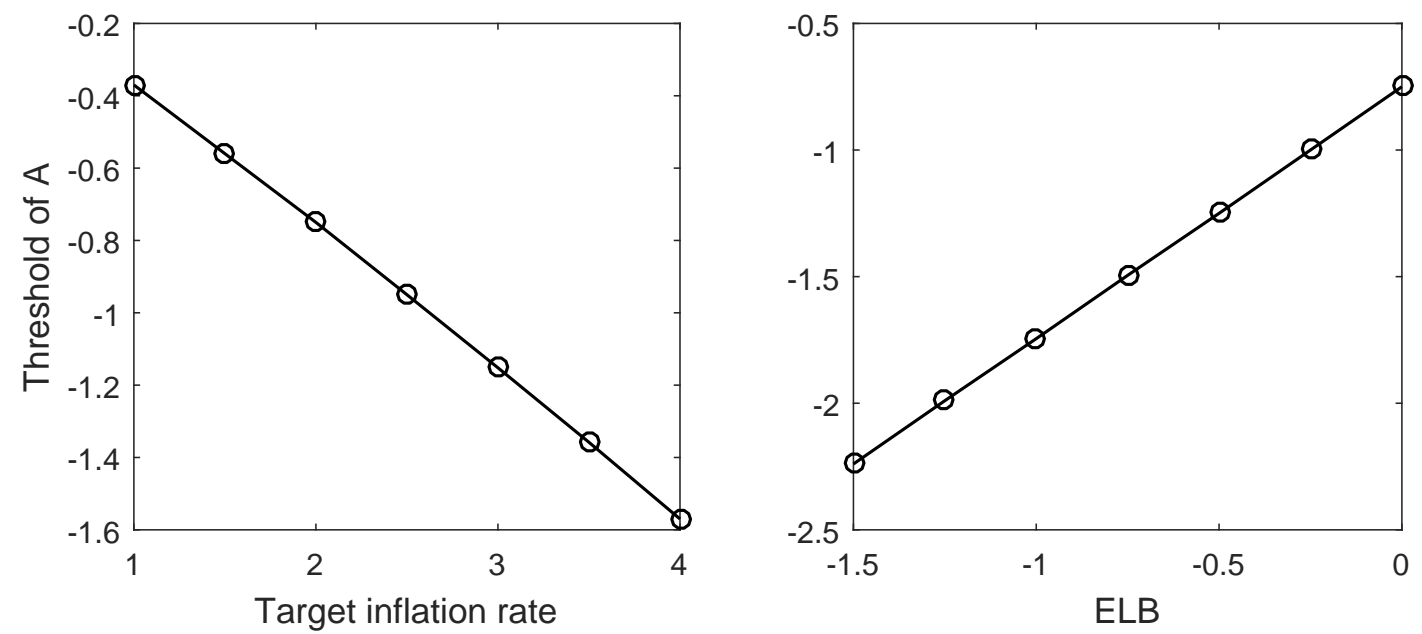

not by directly lowering the real interest rate but by giving rise to the expectation of an accommodative monetary policy stance. For instance, since the increase in $\bar{\pi}$ from $1 \%$ to $2 \%$ implies that the central bank will continue monetary easing even when $\pi_{1}>1 \%$, the increase in $\bar{\pi}$ raising $c_{1}$ and $\pi_{1}$ by changing the expectation of private agents for the monetary policy behavior.

Next, the right panel of Figure 4 shows the result of policy experiments with respect to the value of the ELB, $\kappa$. This policy experiment is motivated by the fact that some central banks have lowered the ELB by introducing a negative interest rate policy to deal with a low inflation environment. In the figure, the horizontal axis depicts the value of $\kappa$, while the vertical axis represents the productivity growth threshold, $A^{*}$. The figure indicates that threshold productivity $A^{*}$ is an increasing function with respect to the value of the ELB, which implies that the central bank can reduce the productivity growth threshold by lowering the value of the ELB. For instance, the figure indicates that the expected growth threshold decreases from $-0.7 \%$ to $-1.3 \%$ if the central bank reduces the value of the ELB from $0.0 \%$ to $-0.5 \%$. The intuition behind this policy effect is simple: under a lower ELB, the probability of reaching the ELB is reduced and the increase of the real interest gap at the ELB is mitigated. The result of this 
policy experiment suggests that a negative interest rate policy may help to raise longrun inflation - a finding that stands in contrast with the results of previous studies. For example, as mentioned earlier, Benhabib et al. (2002) construct a model with multiple equilibria at different levels of inflation, but because their model does not incorporate any equilibrium selection mechanism, it does not provide any theoretical support for the claim that lowering the ELB through a negative interest rate policy can help to get the economy out of the equilibrium dynamics around a deflationary steady state. Note, however, that this quantitative exercise does not take into account any side effects of the negative interest rate policy including negative effects on financial intermediaries.

In sum, the model presented here implies that equilibrium selection between the inflation equilibrium and the zero-inflation equilibrium takes place depending on the expected productivity growth rate and that the central bank can decrease the productivity growth threshold by (i) raising target inflation or (ii) lowering the ELB, and consequently prevent the economy from moving into the zero-inflation equilibrium even in the face of low productivity growth. Therefore, the policy analysis in this subsection implies that if the prolonged low inflation recently observed in developed countries corresponds to the zeroinflation equilibrium of the model, monetary policy measures such as increasing target inflation or introducing a negative interest rate policy can help to end such prolonged low inflation.

\section{Concluding Remarks}

This study presented a simple two-period general equilibrium model in which price competition among firms is described as a coordination game. While the model has multiple Nash equilibria with different levels of inflation due to the existence of the ELB and menu costs, equilibrium selection based on global games implies that if there is a small amount of uncertainty with regard to economic fundamentals, equilibrium selection between the inflation equilibrium and the zero-inflation equilibrium takes place depending on the expected productivity growth rate. This result contrasts with previous studies in the monetary economics literature. Finally, the quantitative policy analysis indicated 
that monetary policy measures such as an increase in the target inflation rate and the introduction of a negative interest rate policy can prevent the economy from moving into the zero-inflation equilibrium. This result suggests that such policies may help to end the prolonged period of low inflation recently observed in developed countries.

A number of avenues for future research remain. First, while this study focused on a simple two-period model for the sake of a better understanding of the theoretical implications, it should be extended to an infinite horizon model with rational expectations in order to obtain more realistic policy implications. Second, another important next step is the empirical examination of the results obtained in this study. In particular, whether changes in the target rate of inflation observed in some countries have been effective is an important empirical issue that could help to test the theoretical implications of the model developed here. These are interesting and important questions that are left for future research.

\section{References}

Benhabib, J., Schmitt-Grohe, S., and Uribe, M. (2002). Avoiding Liquidity Traps. Journal of Political Economy, 110(3):535-563.

Christiano, L., Eichenbaum, M., and Rebelo, S. (2011). When Is the Government Spending Multiplier Large? Journal of Political Economy, 119(1):78 - 121.

Fernandez-Villaverde, J., Guerron-Quintana, P., and Rubio-Ramirez, J. F. (2014). Supply-Side Policies and the Zero Lower Bound. IMF Economic Review, 62(2):248260.

Goldstein, I. and Pauzner, A. (2005). Demand-Deposit Contracts and the Probability of Bank Runs. Journal of Finance, 60(3):1293-1327.

Iacoviello, M. and Michelis, A. D. (2016). Raising an Inflation Target: The Japanese Experience with Abenomics. European Economic Review, In Press. 
Kuroda, H. (2016). The Battle Against Deflation: The Evolution of Monetary Policy and Japan's Experience. Speech at columbia university in new york.

Morris, S. and Shin, H. S. (1998). Unique Equilibrium in a Model of Self-Fulfilling Currency Attacks. American Economic Review, 88(3):587-97.

Taylor, J. B. (1999). The robustness and efficiency of monetary policy rules as guidelines for interest rate setting by the European central bank. Journal of Monetary Economics, 43(3):655-679.

\section{Appendix}

This Appendix provides the proofs for Lemma 1, Lemma 2, and Theorem 1.

\section{Proof of Lemma 1}

Firms' profit $\tilde{\Pi}\left(\pi_{i, 1}, \pi_{1}(n) ; A\right)$ can be written as follows:

$$
\tilde{\Pi}\left(\pi_{i, 1}, \pi_{1} ; A\right)=\left\{\begin{array}{ll}
\frac{\theta-1}{\theta \psi} \pi_{i, 1}^{1-\theta} \bar{\pi}^{\phi-1} \pi_{1}^{\theta-\phi}-\frac{1}{\psi}\left(\frac{\theta-1}{\theta}\right)^{2} \pi_{i, 1}^{-\theta} \bar{\pi}^{2(\phi-1)} \pi_{1}^{\theta+2-2 \phi} & \text { if } A \bar{\pi}\left(\frac{\pi_{1}}{\bar{\pi}}\right)^{\phi}>\kappa \\
\frac{\theta-1}{\kappa \theta \psi} A \pi_{i, 1}^{1-\theta} \pi_{1}^{\theta}-\frac{1}{\psi}\left(\frac{\theta-1}{\kappa \theta} A\right)^{2} \pi_{i, 1}^{-\theta} \pi_{1}^{\theta+2} & \text { otherwise }
\end{array} .\right.
$$

The first case represents firms' profit when the ELB does not bind, while the second case represents the profit when the does ELB bind. Since (i) $\pi_{1}(n)$ is monotonically increasing with respect to $n$, and (ii) the profit function is continuous with respect to $\pi_{1}$ and $A$, what needs to be shown is that $u\left(A, \pi_{1}\right) \equiv \tilde{\Pi}\left(\bar{\pi}, \pi_{1} ; A\right)-\tilde{\Pi}\left(1, \pi_{1} ; A\right)$ is increasing with respect to $\pi_{1}$ and $A$ both in non-ELB states and at the ELB.

First, it is shown that $\partial u / \partial \pi_{1} \geq 0$. When $A \bar{\pi}\left(\pi_{1} / \bar{\pi}\right)^{\phi}>\kappa$,

$$
\begin{aligned}
\frac{\partial u}{\partial \pi_{1}}>0 \Leftrightarrow & \quad\left[\frac{(\theta-1)(\theta-\phi)}{\theta \psi} \bar{\pi}^{\phi-\theta} \pi_{1}^{\theta-\phi-1}-\frac{1}{\psi}\left(\frac{\theta-1}{\theta}\right)^{2}(\theta+2-2 \phi) \bar{\pi}^{2(\phi-1)-\theta} \pi_{1}^{\theta+1-2 \phi}\right] \\
& -\left[\frac{(\theta-1)(\theta-\phi)}{\theta \psi} \bar{\pi}^{\phi-1} \pi_{1}^{\theta-\phi-1}-\frac{1}{\psi}\left(\frac{\theta-1}{\theta}\right)^{2}(\theta+2-2 \phi) \bar{\pi}^{2(\phi-1)} \pi_{1}^{\theta+1-2 \phi}\right]>0 \\
& \Leftrightarrow \frac{\theta+2-2 \phi}{\theta-\phi} \bar{\pi}^{\phi-2}>\frac{\bar{\pi}^{\theta-1}-1}{\bar{\pi}^{\theta}-1} \frac{\theta}{\theta-1} .
\end{aligned}
$$


The last inequality is indeed satisfied under condition (13). Also, when $A \bar{\pi}\left(\pi_{1} / \bar{\pi}\right)^{\phi} \leq \kappa$,

$$
\begin{aligned}
\frac{\partial u}{\partial \pi_{1}}>0 \Leftrightarrow & {\left[\frac{\theta-1}{\kappa \psi} A \bar{\pi}^{1-\theta} \pi_{1}^{\theta-1}-\frac{1}{\psi}\left(\frac{\theta-1}{\kappa \theta} A\right)^{2}(\theta+2) \bar{\pi}^{-\theta} \pi_{1}^{\theta+1}\right] } \\
& -\left[\frac{\theta-1}{\kappa \theta \psi} A \pi_{1}^{\theta-1}-\frac{1}{\psi}\left(\frac{\theta-1}{\kappa \theta} A\right)^{2}(\theta+2) \pi_{1}^{\theta+1}\right]>0 \\
\Leftrightarrow & \frac{A(\theta+2)}{\theta \bar{\pi}}\left(\pi_{1}\right)^{2}>\kappa \frac{\bar{\pi}^{\theta-1}-1}{\bar{\pi}^{\theta}-1} \frac{\theta}{\theta-1} \\
& \Leftarrow A>\frac{\theta \kappa \bar{\pi}^{\phi-1}}{\theta+2} .
\end{aligned}
$$

The last inequality is satisfied under condition (14).

Next, it is shown that $\partial u / \partial A \geq 0$. When $A \bar{\pi}\left(\pi_{1} / \bar{\pi}\right)^{\phi}>\kappa$, it is clear that $\partial u / \partial A=0$, since $u(\cdot)$ is independent of $A$. Also, when $A \bar{\pi}\left(\pi_{1} / \bar{\pi}\right)^{\phi} \leq \kappa$,

$$
\begin{aligned}
\frac{\partial u}{\partial A}>0 & \Leftrightarrow\left[\frac{\theta-1}{\kappa \theta \psi} \bar{\pi}^{1-\theta} \pi_{1}^{\theta}-\frac{2 A}{\psi}\left(\frac{\theta-1}{\kappa \theta}\right)^{2} \bar{\pi}^{-\theta} \pi_{1}^{\theta+2}\right]-\left[\frac{\theta-1}{\kappa \theta \psi} \pi_{1}^{\theta}-\frac{2 A}{\psi}\left(\frac{\theta-1}{\kappa \theta}\right)^{2} \pi_{1}^{\theta+2}\right]>0 \\
& \Leftrightarrow \frac{2 A}{\bar{\pi}}\left(\pi_{1}\right)^{2}>\kappa \frac{\bar{\pi}^{\theta-1}-1}{\bar{\pi}^{\theta}-1} \frac{\theta}{\theta-1} \\
& \Leftarrow A>\frac{\kappa \bar{\pi}^{\phi-1}}{2} .
\end{aligned}
$$

Thus, condition (14) implies that the last inequality is satisfied.

\section{Proof of Lemma 2}

It is first shown that there exists an $\underline{A}$ satisfying $u\left(A, \pi_{1}\right)<0$ for all $\pi_{1} \in[1, \bar{\pi}]$ and $A \leq \underline{A}$. Note that when $A<\kappa / \bar{\pi}$, the ELB binds for all $\pi_{1} \in[1, \bar{\pi}]$. Also, since we know that $u\left(A, \pi_{1}\right)$ is increasing with respect to $\pi_{1}$, what needs to be shown is that there is some $\underline{A}$ satisfying $u(A, \bar{\pi})<0$ for all $A \leq \underline{A} \leq \kappa / \bar{\pi}$ under $R_{1}=\kappa$. Inserting $R_{1}=\kappa$ and deleting $c_{1}$ by the Euler equation yields

$$
\begin{aligned}
u(A, \bar{\pi})<0 & \Leftrightarrow\left[\frac{\theta-1}{\kappa \theta \psi} A \bar{\pi}-\frac{1}{\psi}\left(\frac{\theta-1}{\kappa \theta} A\right)^{2} \bar{\pi}^{2}\right]-\left[\frac{\theta-1}{\kappa \theta \psi} A \bar{\pi}^{\theta}-\frac{1}{\psi}\left(\frac{\theta-1}{\kappa \theta} A\right)^{2} \bar{\pi}^{\theta+2}\right]<0 \\
& \Leftrightarrow \frac{A \bar{\pi}}{\kappa}<\frac{\bar{\pi}^{\theta-1}-1}{\bar{\pi}^{\theta}-1} \frac{\theta}{\theta-1} \\
& \Leftarrow A<\frac{\kappa}{\bar{\pi}} .
\end{aligned}
$$

The last line comes from condition (13). Thus, by setting $\underline{A}=\kappa / \bar{\pi}$, the proof for the first half of Lemma 2 is complete. Next, it is shown that there exists an $\bar{A}$ satisfying 
$u\left(A, \pi_{1}\right)>0$ for all $\pi_{1} \in[1, \bar{\pi}]$ and $A \geq \bar{A}$. Note that when $A>\kappa \bar{\pi}^{\phi-1}$, the ELB does not bind for all $\pi_{1} \in[1, \bar{\pi}]$ and as a result $u\left(A, \pi_{1}\right)$ is independent of $A$. Also, since we know that $u\left(A, \pi_{1}\right)$ is increasing with respect to $\pi_{1}$, what needs to be shown is that $u(A, 1)>0$ for all $A \geq \kappa \bar{\pi}^{\phi-1}$ under $R_{1}=A \bar{\pi}\left(\pi_{1} / \bar{\pi}\right)^{\phi}$. Inserting $R_{1}=A \bar{\pi}\left(\pi_{1} / \bar{\pi}\right)^{\phi}$ and deleting $c_{1}$ by the Euler equation yields

$$
\begin{aligned}
u(A, 1)>0 & \Leftrightarrow\left[\frac{\theta-1}{\theta \psi} \bar{\pi}^{\phi-\theta}-\frac{1}{\psi}\left(\frac{\theta-1}{\theta}\right)^{2} \bar{\pi}^{2(\phi-1)-\theta}\right]-\left[\frac{\theta-1}{\theta \psi} \bar{\pi}^{\phi-1}-\frac{1}{\psi}\left(\frac{\theta-1}{\theta}\right)^{2} \bar{\pi}^{2(\phi-1)}\right]>0 \\
& \Leftrightarrow \bar{\pi}^{\phi-2}-\frac{\bar{\pi}^{\theta-1}-1}{\bar{\pi}^{\theta}-1} \frac{\theta}{\theta-1}>0 .
\end{aligned}
$$

Thus, by setting $\bar{A}=\kappa \bar{\pi}^{\phi-1}$, the proof for the second half of Lemma 2 is complete.

\section{Proof of Theorem 1}

Before going into the proof, some useful notations are defined. First, denote the threshold strategy by $\tilde{\pi}_{i, 1}\left(a_{i} ; A^{\prime}\right)$, where $A^{\prime}$ represents the threshold. The threshold strategy satisfies

$$
\tilde{\pi}_{i, 1}\left(a_{i} ; A^{\prime}\right)=\left\{\begin{array}{ll}
\bar{\pi} & \text { for all } a_{i} \geq A^{\prime} \\
1 & \text { for all } a_{i}<A^{\prime}
\end{array} .\right.
$$

Assume that all other firms adopt the threshold strategy $\tilde{\pi}_{i, 1}\left(a_{i} ; A^{\prime}\right)$. Then, the following two functions are defined. First, with the expected growth rate $A$, the fraction of firms which choose $\pi_{i, 1}=\bar{\pi}$ is defined as $n\left(A, A^{\prime}\right) \in[0,1]$. Specifically, when $\underline{B}+\varepsilon \leq a_{i} \leq \bar{B}-\varepsilon$, the value of $n\left(A, A^{\prime}\right)$ becomes

$$
n\left(A, A^{\prime}\right)=\left\{\begin{array}{cl}
0 & \text { if } A<A^{\prime}-\varepsilon \\
0.5+\left(A-A^{\prime}\right) /(2 \varepsilon) & \text { if } A \in\left[A^{\prime}-\varepsilon, A^{\prime}+\varepsilon\right] \\
1 & \text { if } A>A^{\prime}+\varepsilon
\end{array}\right.
$$

because the signal follows the uniform distribution $U[A-\varepsilon, A+\varepsilon]$. Second, with signal $a_{i}$, the expected net gain from raising prices is defined as $\Gamma\left(a_{i}, A^{\prime}\right)$. When signal $a_{i}$ satisfies $a_{i} \in[\underline{B}+\varepsilon, \bar{B}-\varepsilon]$, the firm subjectively expects that the true value of $A$ follows the uniform distribution $U\left[a_{i}-\varepsilon, a_{i}+\varepsilon\right]$. Therefore, $\Gamma\left(a_{i}, A^{\prime}\right)$ becomes

$$
\Gamma\left(a_{i}, A^{\prime}\right)=\frac{1}{2 \varepsilon} \int_{a_{i}-\varepsilon}^{a_{i}+\varepsilon} u\left(A, n\left(A, A^{\prime}\right)\right) d A
$$


where $u(\cdot)$ is given in the main text. Given these functions, the following lemma is obtained.

Lemma 3 There is a unique $A^{*}$ solving the equation $\Gamma\left(A^{*}, A^{*}\right)=0$.

Proof. Given the signal $a_{i} \in[\underline{B}+\varepsilon, \bar{B}-\varepsilon]$, (16) indicates that the subjective distribution for $n\left(A, A^{\prime}+\Delta A\right)$ in $A \in\left[a_{i}+\Delta A-\varepsilon, a_{i}+\Delta A+\varepsilon\right]$ is the same as that for $n\left(A, A^{\prime}\right)$ in $A \in\left[a_{i}-\varepsilon, a_{i}+\varepsilon\right]$ for small $\Delta A$. Therefore, since $u(A, n)$ is increasing with respect to $A$ as shown in Lemma $1, \Gamma(A, A)$ is increasing with respect to $A \in[\underline{B}+\varepsilon, \bar{B}-\varepsilon]$. Furthermore, we have

$$
\begin{cases}\Gamma\left(a_{i}, A^{\prime}\right)>0 & \text { for all } a_{i} \geq \bar{A}+\varepsilon \text { and } A^{\prime} \\ \Gamma\left(a_{i}, A^{\prime}\right)<0 & \text { for all } a_{i} \leq \underline{A}-\varepsilon \text { and } A^{\prime}\end{cases}
$$

because of Lemma 2 and the assumptions that $\underline{B}<\underline{A}-2 \varepsilon$ and $\bar{B}>\bar{A}+2 \varepsilon$, meaning that there is a unique $A^{*}$ solving the equation $\Gamma\left(A^{*}, A^{*}\right)=0$.

The rest of the proof consists of three steps. The first step is to show that the threshold strategy $\tilde{\pi}_{i, 1}\left(a_{i} ; A^{*}\right)$ forms a symmetric Bayesian Nash equilibrium. When other firms follow $\tilde{\pi}_{i, 1}\left(a_{i} ; A^{\prime}\right)$, the optimal choice for firm $i$ with signal $a_{i}$ is $\pi_{i, 1}\left(a_{i}\right)=\bar{\pi}$ if $\Gamma\left(a_{i}, A^{\prime}\right)>0$ and $\pi_{i, 1}\left(a_{i}\right)=1$ if $\Gamma\left(a_{i}, A^{\prime}\right)<0$. Therefore, $\tilde{\pi}_{i, 1}\left(a_{i} ; A^{*}\right)$ forms a symmetric Bayesian Nash equilibrium if and only if

$$
\begin{aligned}
& \Gamma\left(a_{i}, A^{*}\right)<0 \text { for all } a_{i}<A^{*}, \\
& \Gamma\left(a_{i}, A^{*}\right)>0 \text { for all } a_{i}>A^{*} .
\end{aligned}
$$

Here, $\Gamma\left(a_{i}, A^{\prime}\right)$ is increasing with respect to $a_{i}$ because $u(A, n)$ is increasing with respect to $A$ and $n$, and because $n\left(A, A^{\prime}\right)$ is increasing with respect to $A$. Therefore, $\Gamma\left(A^{*}, A^{*}\right)=$ 0 immediately leads to $(18)$, implying that $\tilde{\pi}_{i, 1}\left(a_{i} ; A^{*}\right)$ forms a symmetric Bayesian Nash equilibrium.

The second step of the proof consists of showing the uniqueness of a symmetric Bayesian Nash equilibrium. That is, it is shown that any strategy $\pi_{1, i}(\cdot)$ which forms a symmetric Bayesian Nash equilibrium must be the threshold strategy $\tilde{\pi}_{i, 1}\left(a_{i} ; A^{*}\right)$. To show the uniqueness, first define $b\left(A^{\prime}\right)$ as the unique $a_{i}$ solving the equation $\Gamma\left(a_{i}, A^{\prime}\right)=0$. 
This is unique because $(17)$ holds and $\Gamma\left(a_{i}, A^{\prime}\right)$ is increasing with respect to $a_{i}$. Then, define $\tilde{b}^{k}\left(A^{\prime}\right)$ as

$$
\tilde{b}^{k}\left(A^{\prime}\right) \equiv\left\{\begin{array}{cc}
A^{\prime} & \text { if } k=0 \\
b\left(\tilde{b}^{k-1}\left(A^{\prime}\right)\right) & \text { if } k \geq 1
\end{array} .\right.
$$

Given the definition of $\tilde{b}^{k}\left(A^{\prime}\right)$, the following lemma is posited.

Lemma 4 Any strategy $\pi_{1, i}(\cdot)$ which forms a symmetric Bayesian Nash equilibrium satisfies

$$
\pi_{i, 1}\left(a_{i}\right)= \begin{cases}\bar{\pi} & \text { if } a_{i}>\tilde{b}^{k-1}(\bar{A}+\varepsilon) \\ 1 & \text { if } a_{i}<\tilde{b}^{k-1}(\underline{A}-\varepsilon)\end{cases}
$$

for all $k \geq 1$. Also, $\lim _{k \rightarrow \infty} \tilde{b}^{k-1}(\bar{A}+\varepsilon)=\lim _{k \rightarrow \infty} \tilde{b}^{k-1}(\underline{A}-\varepsilon)=A^{*}$.

Proof. The proof is by mathematical induction. For $k=1$, (19) obviously holds because of (17). Next, assume that (19) holds for $k=m$. This yields $\Gamma\left(a_{i}, \tilde{b}^{m-1}(\bar{A}+\varepsilon)\right)>0$ for all $a_{i}>\tilde{b}^{m}(\bar{A}+\varepsilon)$ because $\tilde{b}^{m}(\bar{A}+\varepsilon)$ solves $\Gamma\left(a_{i}, \tilde{b}^{m-1}(\bar{A}+\varepsilon)\right)=0$ for $a_{i}$ and $\Gamma\left(a_{i}, \tilde{b}^{m-1}(\bar{A}+\varepsilon)\right)$ is increasing with respect to $a_{i}$. Therefore, any $\pi_{i, 1}(\cdot)$ which forms a symmetric Bayesian Nash equilibrium must choose $\pi_{i, 1}\left(a_{i}\right)=\bar{\pi}$ for all $a_{i}>\tilde{b}^{m}(\bar{A}+\varepsilon)$, thus implying that the first line of (19) is satisfied for $k=m+1$. Similarly, it is shown that any $\pi_{i, 1}\left(a_{i}\right)$ which forms a symmetric Bayesian Nash equilibrium must choose $\pi_{i, 1}\left(a_{i}\right)=1$ for all $a_{i}<\tilde{b}^{m}(\underline{A}-\varepsilon)$, and the second line of (19) is satisfied for $k=m+1$. In this way, it can be shown that any strategy $\pi_{1, i}(\cdot)$ which forms a symmetric Bayesian Nash equilibrium satisfies (19) for all $k \geq 1$.

Next, it is shown that $\tilde{b}^{k-1}(\bar{A}+\varepsilon) \rightarrow A^{*}$ as $k \rightarrow \infty$. This statement is equivalent to showing that $A^{*} \leq b(x)<x$ for all $x>A^{*}$ given the definition of $\tilde{b}^{k}\left(A^{\prime}\right)$. We have $A^{*} \leq b(x)$ for all $x>A^{*}$, because, if this is not the case, $\Gamma(b(x), x)=0$ for some $b(x)<A^{*}$ and $x>A^{*}$, which contradicts to Lemma 3. Also, if $x>A^{*}$, we have $\Gamma(x, x)>0$ because of Lemma 3. Thus, we have $b(x)<x$ because $b(x)$ solves $\Gamma\left(a_{i}, x\right)=0$ for $a_{i}$ and $\Gamma\left(a_{i}, A^{\prime}\right)$ is increasing with respect to $a_{i}$. Similarly, it can be shown that $\tilde{b}^{k-1}(\underline{A}-\varepsilon) \rightarrow A^{*}$ as $k \rightarrow \infty$.

At the limit, $k \rightarrow \infty$, this lemma implies that any strategy $\pi_{1, i}(\cdot)$ which forms a symmetric Bayesian Nash equilibrium must be the threshold strategy $\tilde{\pi}_{i, 1}\left(a_{i} ; A^{*}\right)$. 
As the final step of the proof, it is shown that the threshold $A^{*}$ can be computed by solving $\int_{0}^{1} u\left(A^{*}, n\right) d n=0$ when $\varepsilon \rightarrow 0$. Note that $\Gamma\left(A^{*}, A^{*}\right)=0$ is equivalent to,

$$
\int_{A^{*}-\varepsilon}^{A^{*}+\varepsilon} u\left(A, n\left(A, A^{*}\right)\right) d A=0 .
$$

By definition, $n\left(A, A^{*}\right)=0.5+\left(A-A^{*}\right) /(2 \varepsilon)$ for $A \in\left[A^{*}-\varepsilon, A^{*}+\varepsilon\right]$. Thus, a change of variables yields

$$
\int_{0}^{1} u\left(A\left(n, A^{*}\right), n\right) d n=0
$$

where $A\left(n, A^{*}\right)=2 \varepsilon n-\varepsilon+A^{*}$, which implies that when $\varepsilon \rightarrow 0, \Gamma\left(A^{*}, A^{*}\right)=0$ is equivalent to $\int_{0}^{1} u\left(A^{*}, n\right) d n=0$. 